computer time dependencies that are typically of the order $n^{m}$ where $n$ is the number of basis functions and $m$ is 7 or 8 .

\section{CLOSING REMARKS}

This account is presented as a brief overview of the field of QMC for the electronic structure of atoms and molecules. The attempt has been made to provide a broad cross section of references to enable the interested reader to have access to more extended literature than present space limitations permit.

\section{RELATED ARTICLES}

Benchmark Studies on Small Molecules; Density Functional Applications; Density Functional Theory (DFT), Hartree-Fock $(\mathrm{HF})$, and the Self-consistent Field; G2 Theory; Møller-Plesset Perturbation Theory; Path Integral Methods; Pseudospectral Methods in Ab Initio Quantum Chemistry; $r_{12}$-Dependent Wavefunctions.

\section{REFERENCES}

1. B. L. Hammond, W. A. Lester, Jr., and P. J. Reynolds, 'Monte Carlo Methods in Ab Initio Quantum Chemistry', World Scientific, Singapore, 1994.

2. J. M. Hammersley and D. C. Handscomb, 'Monte Carlo Methods', Methuen, London, 1964.

3. D. M. Ceperley, Rev. Mod. Phys., 1995, 67, 279-355.

4. A. Szabo and N. S. Ostlund, 'Modern Quantum Chemistry', MacMillan, New York, 1982.

5. J. B. Anderson, J. Chem. Phys., 1975, 63, 1499-1503.

6. N. Metropolis, A. W. Rosenbluth, M. N. Rosenbluth, A. H. Teller, and E. Teller, J. Chem. Phys., 1953, 21, 1087-1092.

7. P. J. Reynolds, D. M. Ceperley, B. J. Alder, and W. A. Lester, Jr., J. Chem. Phys., 1982, 77, 5593-5603.

8. C. J. Umrigar, Phys. Rev. Lett., 1993, 71, 408-411.

9. R. N. Barnett, Z. Sun, and W. A. Lester, Jr., Chem. Phys. Lett., 1997, 273, 322-328.

10. H.-J. Flad, M. Caffarel, and A. Savin, in 'Advances in Quantum Monte Carlo Methods', ed. W. A. Lester, Jr., World Scientific, Singapore, 1997, pp. 73-98.

11. W. A. Lester, Jr. and B. L. Hammond, Annu. Rev. Phys. Chem., 1990, 41, 283-311.

12. J. B. Anderson, Int. Rev. Phys. Chem., 1995, 14, 85-112.

13. C. J. Umrigar, K. G. Wilson, and J. W. Wilkins, in 'Computer Simulation Studies in Condensed Matter Physics: Recent Developments', eds. D. P. Landau and H. B. Schuttler, Springer, New York, 1988, pp. 185-194.

14. K. E. Schmidt and J. W. Moskowitz, J. Chem. Phys., 1990, 93, 4172-4178.

15. S. F. Boys and N. C. Handy, Proc. R. Soc. London, Ser. A, 1969, 310, 63-78.

16. C. Filippi and C. J. Umrigar, J. Chem. Phys., 1996, 105, 213-226.

17. Z. Sun, S.-Y. Huang, R. N. Barnett, and W. A. Lester, Jr., J. Chem. Phys., 1990, 93, 3326-3332.

18. C. J. Umrigar, M. P. Nightingale, and K. Runge, J. Chem. Phys., 1993, 99, 2865-2890.

19. J. B. Anderson, J. Chem. Phys., 1976, 65, 4121-4127.

20. D. M. Arnow, M. H. Kalos, M. A. Lee, and K. E. Schmidt, J. Chem. Phys., 1982, 77, 5562-5572.

21. D. L. Diedrich and J. B. Anderson, J. Chem. Phys., 1994, 100, 8089-8095.

22. J. B. Anderson, C. A. Traynor, and B. M. Boghosian, J. Chem. Phys., 1993, 99, 345-351.
23. B. Chen and J. B. Anderson, J. Chem. Phys., 1995, 102, 4491-4494.

24. D. M. Ceperley and B. J. Alder, Phys. Rev. Lett., 1980, 45. 566-569.

25. D. M. Ceperley and B. J. Alder, J. Chem. Phys., 1984, 81. 5833-5844.

26. M. Caffarel, F. X. Gadea, and D. M. Ceperley, EuroPhys. Lett., 1991, 16, 249-254.

27. M. Caffarel and D. M. Ceperley, J. Chem. Phys., 1992, 97, 8415-8423.

28. R. N. Barnett and W. A. Lester, Jr., in 'Computational Chemistry: Review of Current Trends', ed. J. Leszczynski, World Scientific, Singapore, 1997, pp. 125-139.

29. R. M. Grimes, B. L. Hammond, P. J. Reynolds, and W. A. Lester, Jr., J. Chem. Phys., 1986, 85, 4749-4750.

30. A. Lüchow and J. B. Anderson, J. Chem. Phys., 1996, 105, $7573-7578$.

31. R. N. Barnett, P. J. Reynolds, and W. A. Lester, Jr., J. Comput. Phys., 1991, 96, 258-276.

32. J. Casulleras and J. Boronat, J. Phys. Rev. B, 1995, 52, 3654-3661.

33. Z. Sun, R. N. Barnett, and W. A. Lester, Jr., J. Chem. Phys., 1992, 96, 2422-2423.

34. B. L. Hammond, P. J. Reynolds, and W. A. Lester, Jr., J. Chem. Phys., 1987, 87, 1130-1136.

35. M. M. Hurley and P. A. Christiansen, J. Chem. Phys., 1987, 86, $1069-1070$.

36. C. Greeff, W. A. Lester, Jr., and B. L. Hammond, in 'Advances in Quantum Monte Carlo Methods', ed. W. A. Lester, Jr., World Scientific, Singapore, 1997, pp. 117-131.

37. D. M. Ceperley, J. Stat. Phys., 1996, 43, 815-826.

38. L. Mitas, E. L. Shirley, and D. M. Ceperley, J. Chem. Phys., 1991, 95, 3467-3475.

39. D. E. Woon and T. H. Dunning, Jr., J. Chem. Phys., 1994, 101, 8877-8893.

40. C. W. Bauschlicher, H. Partridge, S. R. Langhoff, P. R. Taylor, and S. P. Walch, J. Chem. Phys., 1987, 86, 7007-7012.

41. Z. Fu, G. W. Lemire, G. A. Bishea, and M. D. Morse, J. Chem. Phys., 1990, 93, 8420-8441.

42. C. Moore, 'Atomic Energy Levels', Vol. 1, National Bureau of Standards, Washington, DC, 1949.

43. H. Hotop and W. C. Lineberger, J. Phys. Chem. Ref. Data, $1985,14,731-750$.

44. R. O. Jones, J. Chem. Phys., 1993, 99, 1194-1206.

45. J. E. Harrington and J. C. Weisshar, J. Chem. Phys., 1990, 93, 854-855.

\section{Monte Carlo Simulations for Complex Fluids}

\section{Berend Smit}

Universiteit van Amsterdam, The Netherlands

1 Introduction

2 Simulation Techniques

3 Applications

4 Concluding Remarks

5 Related Articles

6 References 


\section{INTRODUCTION}

Since the work of van der Waals it has been known that long-range attractions and short-range repulsions between spherical particles are sufficient to explain a vapor-liquid phase transition. Computer simulations have shown that shortrange repulsions between spherical particles are responsible for freezing and melting. 'Since in real fluids in which the atoms resemble spherical particles that have short-range repulsions and long-range attractions one also can observe these three phases (gas, liquid, and solid), such a fluid is often called a simple fluid.

There are, however, a significant number of systems that can not be described using simple spherical particles with longrange attractions. Typical examples are elongated molecules that can form liquid crystalline phases or surfactants that can form various sorts of micelles or vesicles. Compared to simple fluids, the phase behavior of these fluids can be very complicated and therefore these fluids are often called complex fluids.

Since the conventional simulation techniques such as molecular dynamics (see Molecular Dynamics and Hybrid Monte Carlo in Systems with Multiple Time Scales and Long-range Forces: Reference System Propagator Algorithms; Molecular Dynamics: Simulations of Nucleic Acids; Molecular Dynamics: Studies of Lipid Bilayers; and Molecular Dynamics: Techniques and Applications to Proteins) and the Monte Carlo technique (see Monte Carlo Simulations for Liquids and Monte Carlo Simulations for Polymers) are equally valid for simple and complex fluids, one may wonder whether a simulation of a complex system is simply changing the force fields and potential. In some cases it may indeed be as simple as that, in particular if the computers are powerful enough to deal with these more complex systems in a reasonable amount of time. For some problems, however, the increase in computer time can be prohibitively large; it may take many years of super-computer time before a calculation is finished. For these types of system it is important that novel algorithms will be developed.

Let us illustrate the above with a specific example. Although this example is strongly application driven, the conclusions are equally valid for more fundamental problems in simulations of complex fluids. There is considerable interest in studying the adsorption of alkanes in the pores of a zeolite. Zeolites are microporous materials which are used as catalysts in petrochemical applications (see Zeolites: Applications of Computational Methods). A prerequisite for an understanding of the catalytic activity of these zeolites is a knowledge of the behavior of the molecules adsorbed in their narrow pores. Since this type of information is extremely difficult to obtain experimentally, simulations appear to be an attractive alternative. ${ }^{2}$ Indeed, over the last decade many simulation studies on the behavior of molecules in zeolites thave been published (for a review see Ref. 3). A more careful look at these studies reveals that most simulations concem the adsorption of noble gases or methane, only a few studies of ethane or propane have been published. In petrochemical applications of zeolites, however, we are interested in the behavior of much longer alkanes such as octane and decane.

The reason why only small molecules have been studied becomes clear from the work of June et al. ${ }^{4}$ and Hernández and Catlow, ${ }^{\mathbf{5}}$ in which molecular dynamics simulations were used to investigate the diffusion of butane and hexane in the zeolite silicalite. June et al. showed that the diffusion of br." he from one channel of the zeolite into another channel is $y$ slow compared with diffusion of bulk butane. As a consequence many hours of super-computer time were required to obtain reliable results. In addition, since the diffusion coefficient decreases significantly with increasing chain length. extrapolation of these results suggests that many years of super-computer time would be required to obtain comparable results for the longer alkanes.

The above example illustrates one of the main limitations of molecular dynamics: in such a simulation, the approach is to mimic the behavior of the molecules as realistically as possible. If successful, all properties will be like in nature, including the diffusion. If the molecules diffuse slowly this will be reflected in very long simulation times and in the case of long chain alkanes these simulation times tum out to be much longer than we can currently afford. In principle, one can circumvent this intrinsically slow dynamics by using a Monte Carlo technique. In a Monte Carlo simulation one does not have to follow the 'natural path' and one can, for example, perform a move in which it is attempted to displace a molecule to a random position in the zeolite. If such a move is acc 1 , it corresponds to a very large jump in phase space.

Again, utilization of this type of unnatural Monte Carlo move turns out to be limited to small molecules. For example, Goodbody et al. ${ }^{6}$ have used this Monte Carlo trick to determine the adsorption isotherms of methane in a zeolite. In such a simulation one can observe that out of the 1000 attempts to move a methane molecule to a random position in the zeolite999 attempts will be rejected because the methane molecule overlaps with a zeolite atom. If we were to perform a similar move with an ethane molecule, we would need $1000 \times 1000$ attempts to have one that was successful. Clearly, this random insertion scheme will break down for any but the smallest alkanes.

The above example, the adsorption of chain molecules in the pores of a zeolite, is used to illustrate the problems that may occur if one uses conventional simulation techniques for more complex systems. Similar problems may occur in the simulation of phase equilibria of chain molecules, simulations of polymers, or liquid crystals. For many of these systems it is relatively straightforward to implement the force fialds to simulate these systems; however, the simulation is required to determine reliable equilibrium properties may be prohibitively long. These simulation times may even be so extreme that it cannot be expected that increasing computer power will be of any help. To be able to perform simulations on complex systems it is therefore important to develop novel algorithms that are orders of magnitude more efficient than the conventional algorithms. In this article such algorithms are discussed.

\section{SIMULATION TECHNIQUES}

To make Monte Carlo moves of long chain molecules possible, Siepmann and Frenkel ${ }^{7}$ developed the configurational-bias Monte Carlo technique for lattice models. This technique is based on the early work of Rosenbluth and Rosenbluth ${ }^{8}$ and Harris and Rice. ${ }^{9}$ This technique has since been extended to continutum models by Frenkel et al. ${ }^{10}$ and de Pablo et al. ${ }^{11}$ 
The starting point for configurational-bias Monte Carlo is the scheme as introduced by Rosenbluth and Rosenbluth in $1954 .^{8}$ At first sight, this may not appear to lead to a new algorithm but to a very old one. The original Rosenbluth scheme itself was designed as a method to sample polymer conformations. However, it suffers from the drawback that it generates an unrepresentative sample of all polymer conformations, i.e., the probability of generating a particular conformation using the Rosenbluth scheme is not proportional to its Boltzmann weight. Rosenbluth and Rosenbluth introduced the weight $W$ to correct for this biasing in the computation of thermal averages. Batoulis and Kremer ${ }^{12}$ have shown that this correction procedure only works for relatively short chains. The solution of this problem is to bias the Rosenbluth sampling in such a way that the correct (Boltzmann) distribution of chain conformations is generated in a Monte Carlo sequence. In the configurational-bias scheme, the Rosenbluth weight is used to bias the acceptance of trial conformations that are generated with the Rosenbluth procedure. If one were to use the ordinary Metropolis acceptance rule, such a bias in the configurations of the molecules would lead to an incorrect distribution of configurations. Frenkel et al, ${ }^{10}$ have shown that by using acceptance with the Rosenbluth weight this bias can be removed exactly. As a consequence, all conformations are generated with their correct Boltzmann weight. This removes the main drawback of the original Rosenbluth scheme.

\subsection{Beyond Metropolis}

The general idea of biased sampling is best explained by considering a simple example. Let us assume that we have developed a Monte Carlo scheme that allows us to generate trial configurations with a probability that depends on the potential energy of that configuration:

$$
\alpha(o \rightarrow n)=f[U(n)]
$$

For the reverse move, we have

$$
\alpha(n \rightarrow 0)=f[\mathcal{U}(0)]
$$

Suppose we want to sample the N, V, T ensemble, which implies that we have to generate configurations with a Boltzmann distribution:

$$
\mathcal{N}(\Omega) \propto \exp [-\beta \mathcal{U}(n)]
$$

To prove that the correct distribution is sampled, we have to demonstrate that detailed balance is obeyed

$$
K(\mathrm{o} \rightarrow \mathrm{n})=K(\mathrm{n} \rightarrow \mathrm{o})
$$

where $K(\mathrm{o} \rightarrow \mathrm{n})$ is the flow of configuration o to $n$. This flow is given by the product of the probability of being in configuration $o$, the probability of generating configuration $n$, and the probability of accepting this move,

$$
K(\mathrm{o} \rightarrow \mathrm{n})=\mathcal{N}(\mathrm{o}) \times \alpha(\mathrm{o} \rightarrow \mathrm{n}) \times \operatorname{acc}(\mathrm{o} \rightarrow \mathrm{n})
$$

If we impose detailed balance, we get as a condition for the acceptance rule,

$$
\frac{\operatorname{acc}(0 \rightarrow n)}{\operatorname{acc}(n \rightarrow 0)}=\frac{f[\mathcal{U}(\mathrm{n})]}{f[\mathcal{U}(0)]} \exp \{-\beta\{\mathcal{U}(\mathrm{n})-\mathcal{U}(0)]\}
$$

A possible acceptance rule that obeys this condition is

$$
\operatorname{acc}(0 \rightarrow n)=\min \left(1, \frac{f\{\mathcal{U}(n)\}}{f[\mathcal{U}(0)]} \exp \{-\beta\{\mathcal{U}(n)-\mathcal{U}(0)]\}\right)
$$

This derivation shows that we can introduce an arbitrary biasing function $f(\mathcal{U})$ into the sampling scheme and generate a Boltzmann distribution of configurations, provided that the acceptance rule is modified in such a way that the bias is removed from the sampling scheme. Ideally, by biasing the probability of generating a trial conformation in the right way, we could make the term on the right-hand side of equation (4) always equal to unity. In that case, every trial move will be accepted. Sometimes it is possible to achieve this ideal situation, ${ }^{13}$ however, in general, biased generation of trial moves is simply a technique to enhance the acceptance of such moves without violating detailed balance.

We now illustrate the use of non-Metropolis sampling techniques to demonstrate how they can be used to enhance the efficiency of a simulation of a system containing chain molecules.

\subsection{Configurational-bias Monte Carlo}

\subsubsection{Lattice Models}

The configurational-bias Monte Carlo algorithm consists of the following steps:

1. Generate a trial conformation using the Rosenbluth scheme to grow the entire molecule, or part thereof, and compute its Rosenbluth weight $W(n)$.

2. 'Retrace' the old conformation and determine its Rosenbluth factor, W(o).

3. Accept the trial move with a probability

$$
\operatorname{acc}(0 \rightarrow \mathrm{n})=\min [1, \mathrm{~W}(\mathrm{n}) / \mathrm{W}(\mathrm{o})]
$$

The generation of a trial conformation $n$ of a polymer consisting of $\ell$ monomers is generated using an algorithm based on the method of Rosenbluth and Rosenbluth (see Figure 1):

1. The first atom is inserted at random, and its energy is denoted by $u_{1}(\mathrm{n})$, and the Rosenbluth weight of the first segment by $w_{1}(n)=k \exp \left[-\beta u_{1}(n)\right]$, where $k$ is the coordination number of the lattice, for example, $k=6$ for a simple cubic lattice. (The factor $k$ in the definition of the Rosenbluth weight of the first segment, strictly speaking, is unnecessary. We introduce it here only to make the subsequent notation more compact.)

2. For the next segment, with index $i$, there are $k$ possible trial directions. The energy of trial direction $j$ is denoted by $u_{i}(j)$. From the $k$ possible directions, we select one, say, $n$, with a probability

$$
p_{i}(n)=\frac{\exp \left[-\beta u_{i}(n)\right]}{w_{i}(n)}
$$

where $w_{i}(n)$ is defined as

$$
w_{i}(n)=\sum_{j=1}^{k} \exp \left[-\beta u_{i}(j)\right]
$$

The interaction energy $u_{i}(j)$ includes all interactions of segment $i$ with other molecules in the system and with 

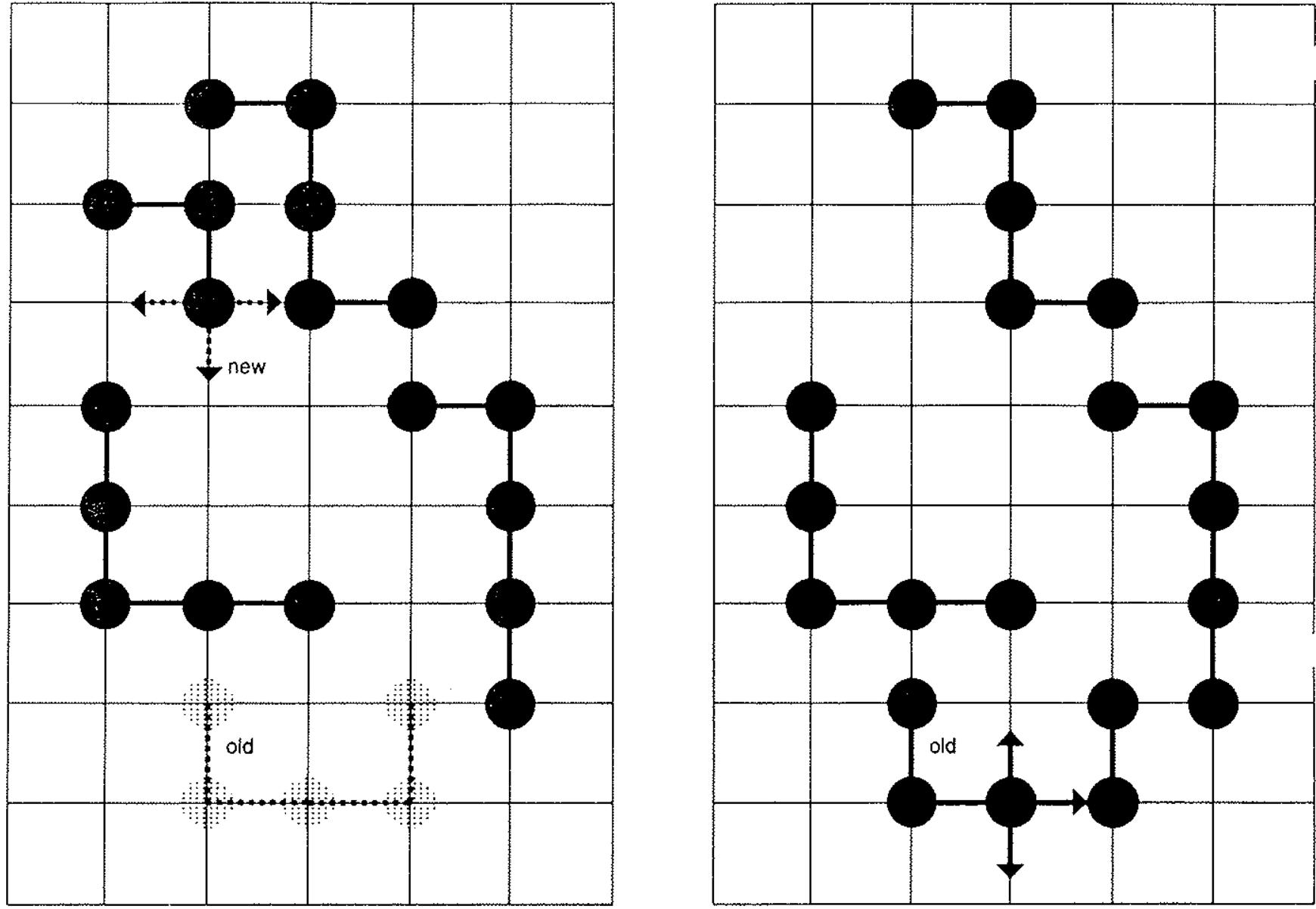

Figure 1 Sketch of the configurational-bias Monte Carlo scheme. The left figure shows the generation of a new configuration and the right figure shows the retracing of the old conformation. The arrows indicate the three trial positions

segments 1 through $i-1$ of the same molecule. It does not include the interactions with segments $i+1$ to $\ell$. Hence, the total energy of the chain is given by $\mathcal{U}(\mathrm{n})=$ $\sum_{i=1}^{\ell} u_{i}(\mathrm{n})$.

3. Step 2 is repeated until the entire chain is grown and we can determine the Rosenbluth factor of configuration $n$ :

$$
\mathrm{W}(\mathrm{n})=\prod_{i=1}^{\ell} \mathrm{w}_{i}(\mathrm{n})
$$

To determine the Rosenbluth factor of the old configuration, $\mathrm{W}(\mathrm{o})$, we use the following steps (see Figure 1).

1. One of the chains, say $o$, is selected at random.

2. We compute the energy of the first monomer $u_{1}(0)$ and its Rosenbluth factor $w_{1}(0)=k \exp \left[-\beta u_{1}(0)\right]$.

3. To compute the Rosenbluth weight for the remainder of the chain, we determine the energy of monomer $i$ at its actual position, and also the energy it would have had it been placed in any of the other $k-1$ sites neighboring the actual position of monomer $i-1$ (see Figure 1 ). These energies are used to calculate

$$
\mathrm{w}_{i}(0)=\exp \left[-\beta u_{i}(0)\right]+\sum_{j=2}^{k} \exp \left[-\beta u_{i}(j)\right]
$$

4. Once the entire chain has been retraced, we determine its Rosenbluth factor:

$$
W(0)=\prod_{i=1}^{l} w_{i}(0)
$$

Finally the trial move from o to $\mathrm{n}$ is accepted with a probability given by

$$
\operatorname{acc}(0 \rightarrow n)=\min [1, W(n) / W(o)]
$$

We now have to demonstrate that the acceptance rule (lu) correctly removes the bias introduced by using equation ( 6 ) of generating new segments in the chain.

The probability of generating a particular conformation $n$ follows from the repetitive use of equation (6):

$$
\alpha(0 \rightarrow n)=\prod_{i=1}^{\ell} \frac{\exp \left[-\beta u_{i}(n)\right]}{w_{i}(n)}=\frac{\exp [-\beta \mathcal{U}(n)]}{W(n)}
$$

Similarly, for the reverse move,

$$
\alpha(\mathrm{n} \rightarrow 0)=\frac{\exp [-\beta U(0)]}{\mathrm{w}(0)}
$$

The requirement of detailed balance (2) imposes the following condition on the acceptance criterion

$$
\frac{\operatorname{acc}(0 \rightarrow n)}{\operatorname{acc}(n \rightarrow 0)}=\frac{W(n)}{W(0)}
$$


Clearly, the proposed acceptance criterion (10) satisfies this condition.

It should be stressed that the value of factor $W(0)$ depends on the direction in which the old configuration is retraced: if we start from monomer 1, we find a different numerical value for $W(0)$ than if we start from monomer $\ell$. As a consequence the probability of such a move depends on the way the factor $W(0)$ has been calculated. Although such a dependence is at first sight counterintuitive, both ways of retracing the old conformation - starting with monomer I or with monomer $\ell$ - result in the correct distribution of states, as long as both ways occur with equal probability during the simulation. This is automatically satisfied in the case of linear chains of identical segments where the labeling of the terminal groups is completely arbitrary.

\subsubsection{Off-lattice Case}

Next we consider configurational-bias Monte Carlo for offlattice systems. An important point that we have to consider is the way in which trial conformations of a chain molecule are generated. In a lattice model, the number of trial conformation is dictated by the lattice. In an off-lattice system, one could generate trial segments with orientations distributed uniformly on a unit sphere. However, for many models of interest this procedure is not very efficient, in particular when there are strong intramolecular interactions (e.g., bending and torsion potentials). The efficiency of a configurational-bias Monte Carlo algorithm depends to a large extent on the method used of generating the trial orientations. For example, an isotropic distribution of trial directions is well suited for completely flexible chains. In contrast, for a stiff chain (e.g., liquid-crystal forming polymer), such a trial position will almost always be rejected because of the intramolecular interactions.

From the preceding discussion, it follows that the intramolecular interactions should be taken into account in generating the set of trial conformations. Here, we consider the case of a Rexible molecule with contributions to the internal energy due to bond bending and torsion. The fully flexible case then follows trivially. Consider a chain of $\ell$ linear segments, the potential energy of a given conformation $\mathcal{U}$ has two contributions:

1. The bonded potential energy $\mathcal{U}^{\text {bond }}$ is equal to the sum of the contributions of the individual joints. A joint between segments $i$ and $i+1$ (say) has a potential energy $u_{i}^{\text {bond }}$ that depends on the angle $\theta$ between the successive segments. For instance, $u_{i}^{\text {bond }}(\theta)$ could be of the form $u_{i}^{\text {bond }}(\theta)=k_{\theta}(\theta-$ $\left.\theta_{0}\right)^{2}$. For realistic models for polyatomic molecules, $u_{i}^{\text {bond }}$ includes all local bonded potential energy changes due to the bending and torsion of the bond from atom $i-1$ to atom $i$.

2. The external potential energy $\mathcal{U}^{\text {ext }}$ accounts for all interactions with other molecules and for all the nonbonded intramolecular interactions. In addition, interactions with any external field that may be present are also included in $\mathcal{U}^{\text {ext }}$.

In what follows we shall denote a chain in the absence of the external interactions as the ideal chain. Note that this is a purely fictitious concept, as real chains always have nonbonded intramolecular interactions.
To perform a configurational-bias Monte Carlo move, we apply the following 'recipe' to construct a conformation of a chain of $\ell$ segments. The construction of chain conformations proceeds segment by segment. Let us consider the addition of one such segment. To be specific, let us assume that we have already grown $i-1$ segments and are trying to add segment $i$. This is done in two steps. First we generate a trial conformation $n$, next we consider the old conformation $o$. A trial conformation is generated as follows:

1. Generate a fixed number (say $k$ ) trial segments. The orientations of the trial segments are distributed according to the Boltzmann weight associated with the bonded interactions of monomer $i\left(u_{i}^{\text {bond }}\right)$. We denote this set of $k$ different trial segments by

$$
\{b\}_{k}=\left\{b_{1} \ldots b_{k}\right\}
$$

where the probability of generating a trial segment $b$ is given by

$$
\begin{aligned}
p_{i}^{\text {bond }}(b) \mathrm{d} b & =\frac{\exp \left[-\beta u_{i}^{\text {bond }}(b)\right] \mathrm{d} b}{\int \mathrm{d} b \exp \left[-\beta u_{i}^{\text {bond }}(b)\right]} \\
& =C \exp \left[-\beta u_{i}^{\text {bond }}(b)\right] \mathrm{d} b
\end{aligned}
$$

2. For all $k$ trial segments, we compute the extemal Boltzmann factors $\exp \left[-\beta u_{i}^{\text {ext }}\left(b_{i}\right)\right]$, and out of these, we select one, denoted by $n$, with a probability

$$
p_{i}^{\text {ext }}\left(b_{\mathrm{n}}\right)=\frac{\exp \left[-\beta u_{i}^{\text {ext }}\left(b_{\mathrm{n}}\right)\right]}{\mathrm{w}_{i}^{\mathrm{ext}}(\mathrm{n})}
$$

where we have defined

$$
\mathrm{w}_{i}^{\mathrm{ext}}(\mathrm{n})=\sum_{j=1}^{k} \exp \left[-\beta u_{i}^{\mathrm{ext}}\left(b_{j}\right)\right]
$$

3. The selected segment $\mathrm{n}$ becomes the $i$ th segment of the trial conformation of the chain.

4. When the entire chain is grown, we calculate the Rosenbluth factor of the chain:

$$
W^{\text {ext }}(n)=\prod_{i=1}^{\ell} w_{i}^{e x t}(n)
$$

where Rosenbluth factor of the first monomer is defined by

$$
\mathrm{w}_{1}^{\mathrm{ext}}(\mathrm{n})=k \exp \left[-\beta u_{1}^{\mathrm{ex}}\left(r_{1}\right)\right]
$$

where $r_{1}$ is the position of the first monomer.

For the old configuration, a similar procedure for calculating its Rosenbluth factor is used.

1. One of the chains is selected at random. This chain is denoted $o$.

2. The extemal energy of the first monomer is calculated. This energy involves only the external interactions. The Rosenbluth weight of this first monomer is given by

$$
w_{1}^{\text {ext }}(0)=k \exp \left[-\beta u_{1}^{\text {ext }}(0)\right]
$$

3. The Rosenbluth factors of the other $\ell-1$ segments are calculated as follows. We consider the calculation of the 
Rosenbluth factor of segment $i$. We generate a set of $k-1$ orientations with a distribution prescribed by the bonded interactions (14). These orientations, together with the actual bond between segment -1 and $i$, form the set of $k$ orientations $\left(b_{0}, b^{\prime *}\right)$. These orientations are used to calculate the external Rosenbluth factor:

$$
w_{i}^{e x t}(0)=\sum_{j=1}^{k} \exp \left[-\beta u_{i}^{\mathrm{ext}}\left(b_{j}\right)\right]
$$

4. For the entire chain the Rosenbluth factor of the old conformation is defined by

$$
W^{e x t}(0)=\prod_{i=1}^{t} w_{i}^{e x t}(0)
$$

After the new configuration has been generated and the Rosenbluth factor of the old configuration has been calculated the move is accepted with a probability

$$
\operatorname{acc}(0 \rightarrow n)=\min \left[1 . W^{e x t}(n) / W^{\text {ext }}(0)\right]
$$

In Ref. 13 it is demonstrated that this algorithm samples the desired distribution.

\section{APPLICATIONS}

Here the use of the CBMC technique is illustrated with some examples of practical relevance.

\subsection{Adsorption of Alkanes in Zeolites}

In the introduction we have used the adsorption of molecules as an example to illustrate the type of problem one can encounter in simulating systems that exhibit slow diffusion. Smit and Siepmann have used the configurational-bias Monte Carlo technique to study the energetics and siting of alkanes in the zeolites silicalite and mordenite. ${ }^{14,15}$ In these simulations alkane molecules are modeled with a united atom model, i.e., a $\mathrm{CH}_{3}$ and a $\mathrm{CH}_{2}$ group are considered as a single interaction centre and the zeolite is modeled as a rigid crystal. The zeolite-alkane interactions are assumed to be dominated by dispersive interaction with the oxygen atoms, which are described with a Lennard-Jones potential. A closely related technique was used by Maginn et al. ${ }^{16}$ Figure 2 shows that the simulations of Smit and Siepmann and Maginn et al. predict heats of adsorption of the longer chain alkanes in silicalite that are in good agreement with the experimental data and the simulations of Bigot and Peuch. ${ }^{17}$

From the simulations, we can determine the distribution of the alkanes over the channels. In Figure 3 the distribution of butane and dodecane is shown graphically. It is interesting to compare the probability distribution of butane with the one of dodecane. Whereas the plots of butane show an equal density of points in the straight and zig-zag channels, dodecane has a significantly lower probability of being in the zig-zag channels. In a sense, silicalite becomes more and more 'unidimensional' with increasing length of the hydrocarbon.

Simulations can also be used to determine adsorption isotherms. Adsorption isotherms are of practical importance since they give information on the number of molecules adsorbed in the pores of a zeolite as a function of the pressure

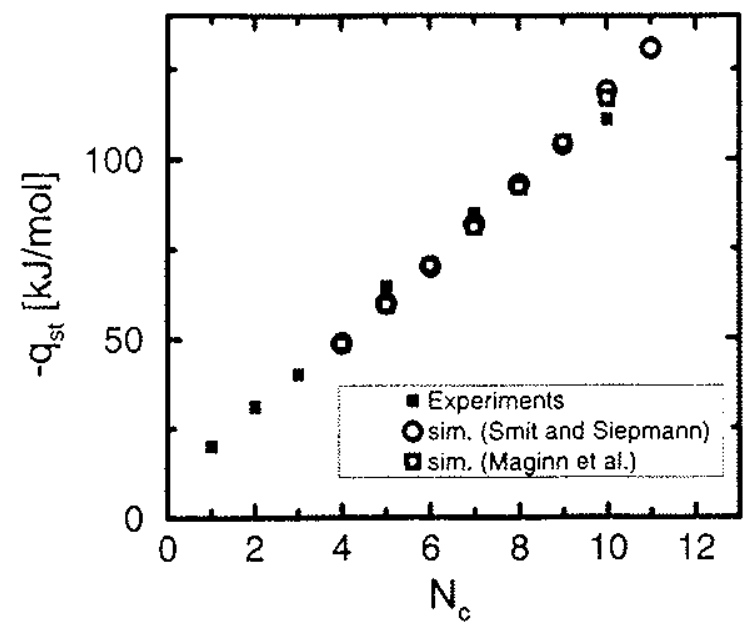

Figure 2 The heats of adsorption of the alkanes in silicalite as a function of the total number of carbon atoms. The closed symbols are experimental and the open symbols are simulation data from Maginn et al. ${ }^{16}$ and Smit and Siepmann ${ }^{14,15}$

of the reservoir. Adsorption isotherms are also of fundame: interest since they may signal phase transitions, such as capillary condensation or wetting, of the fluid inside the pores. ${ }^{18}$ For example, if a system exhibits capillary condensation, one would measure a stepped adsorption isotherm with hysteresis. Steps or kinks without hysteresis are occasionally observed on flat substrates. ${ }^{19}$ Since the pores of most zeolites are of molecular dimension, adsorbed alkane molecules behave like a (pseudo) one-dimensional fluid. In a one-dimensional system phase transitions do not occur and therefore one would expect that for alkanes the adsorption isotherms are of type I, i.e., do not show kinks or steps. If steps occur, they are usually attributed to capillary condensation in the exterior secondary pore system formed by the space between different crystals. ${ }^{20}$ For silicalite, adsorption isotherms have been determined for various $n$-alkanes: for the short-chain alkanes (methane-pentane) the isotherms are indeed of type I, also for decane a type $I$ isothem is observed. For hexane and heptane, however, a kink or step is observed.

Adsorption isotherms are conveniently determined from simulations in the grand-canonical ensemble. In this enseble the temperature and chemical potential are imposed, the number of particles is allowed to fluctuate. Adsorption isotherms of alkanes in silicalite have been simulated by Smit and Maesen. ${ }^{21}$ The simulated adsorption isotherms for hexane and heptane are shown in Figure 4. The agreement of the simulation results with the experimental data is good at high pressures, but at low pressures deviations exist which indicate that the zeolite-alkane model may be further improved. It is interesting to note that for heptane both the experiments and the simulations show a step at approximately half the loading. Also for hexane detailed inspection of the calculated adsorption isotherm shows a kink at this loading. Since the simulations are performed on a perfect single crystal, these deviations from the type I isotherm must be due to a transition of the fluid inside the pores and cannot be attributed to the secondary pore system.

Smit and Maesen attribute this transition to a commensurate 'freezing' in the channels of a zeolite. The length of a hexane molecule is of the order of the length of the period of 

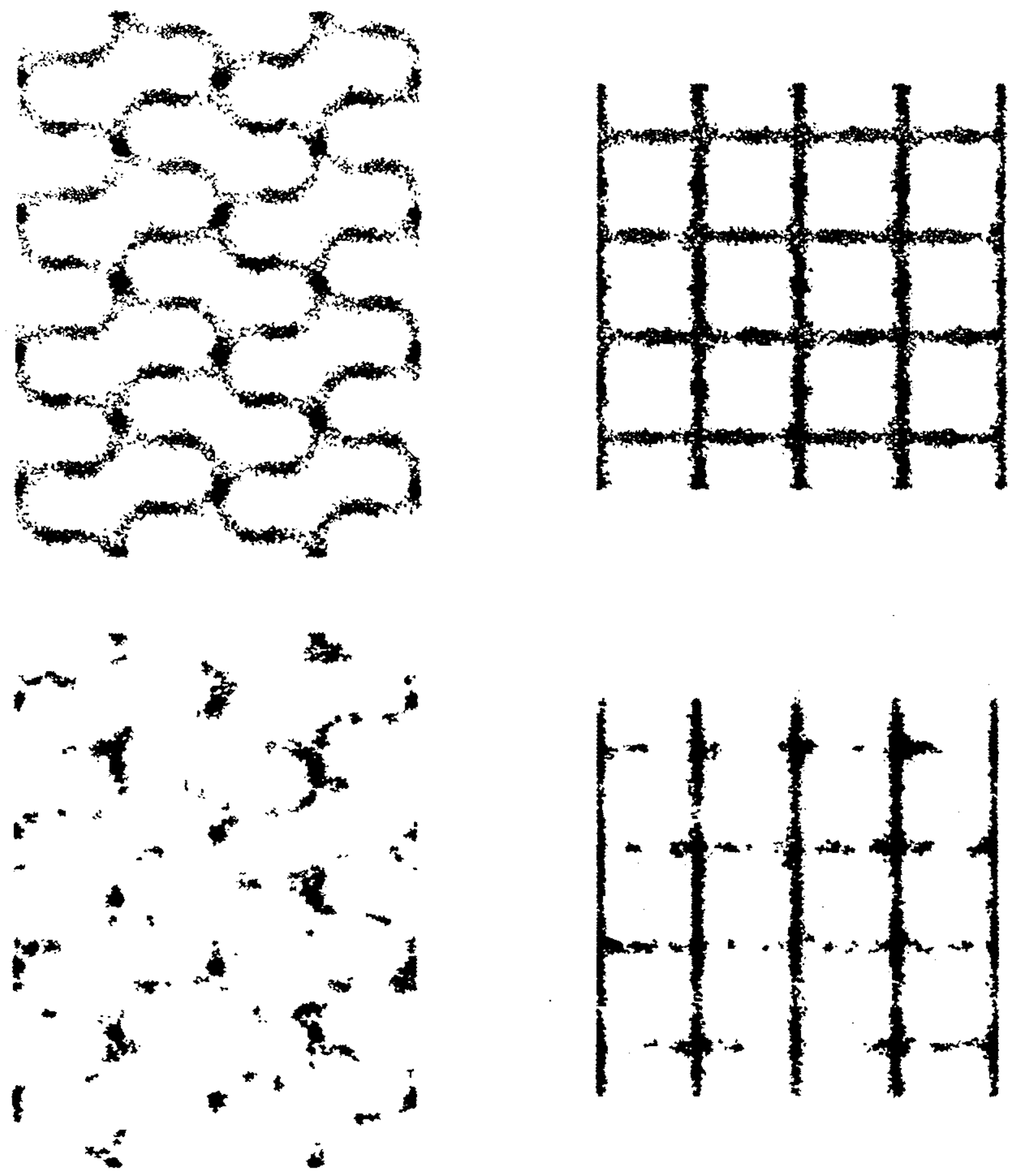

Figure 3 Distribution of butane (top) and dodecane (bottom) over the various channels of silicalite. At regular intervais during the simulations, the end-to-end vector of the alkane molecule is calculated and depending on its orientation a color is selected; blue for the intersection, grey for the zig-zag channel, and magenta for the straight channel, and a dot in the selected color is drawn at the position of the center of mass of the molecule. The density of the dots is a measure of the probability of finding an alkane in a particular section of the zeolite. The left figures are projections on the side plane and the right figures projections on the top plane

the zig-zag channel. At low chemical potential, the hexane molecules move 'freely' in these channels and the molecules will spend part of their time in the intersections. If part of the intersection is occupied, other molecules can not reside in the straight channels at the same time. At high pressures, almost all hexane molecules are exactly fitting into the zigzag channel, they no longer move freely and keep their nose and tail out of the intersection. In such a configuration the entire straight channel can now be tightly packed with hexane molecules (see Figure 5). This may explain the plateau in the 

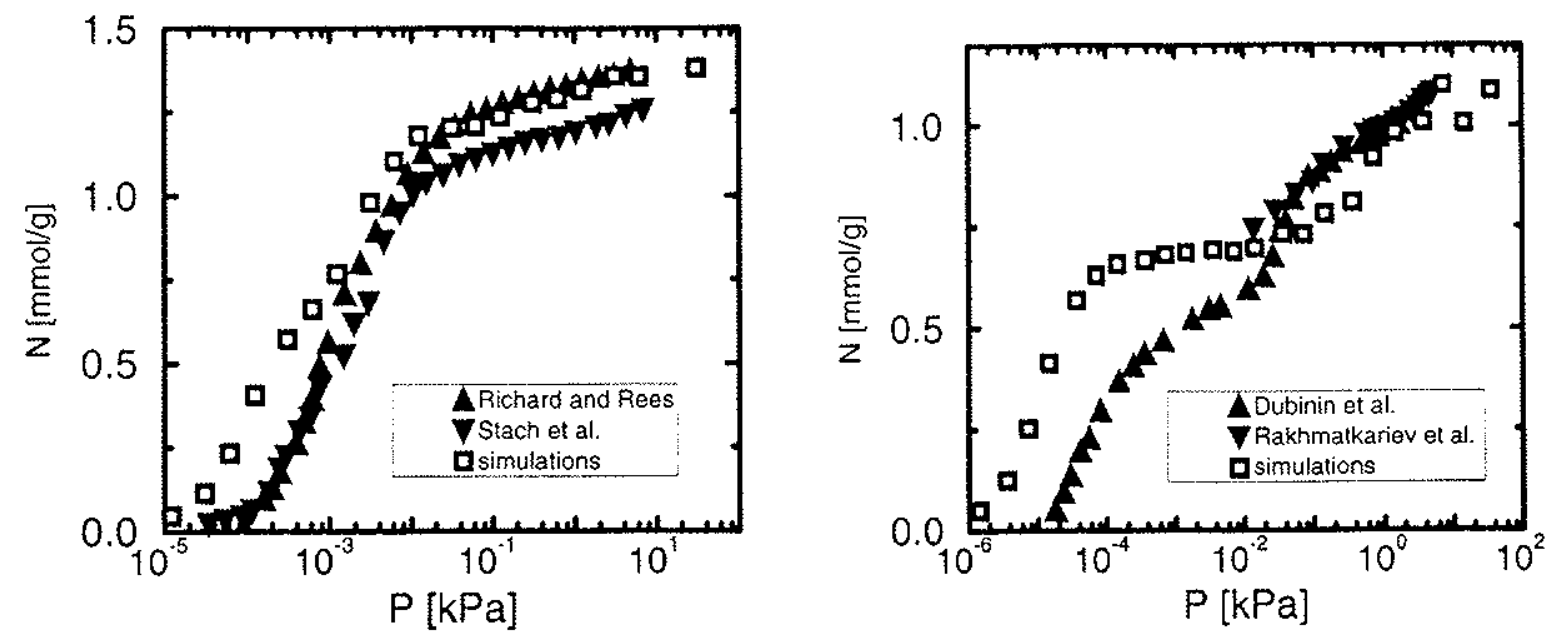

Figure 4 Adsorption isotherms of hexane (left), and heptane (right), the closed symbols are experimental data and the open symbols the results from simulations at $T=298 \mathrm{~K}$
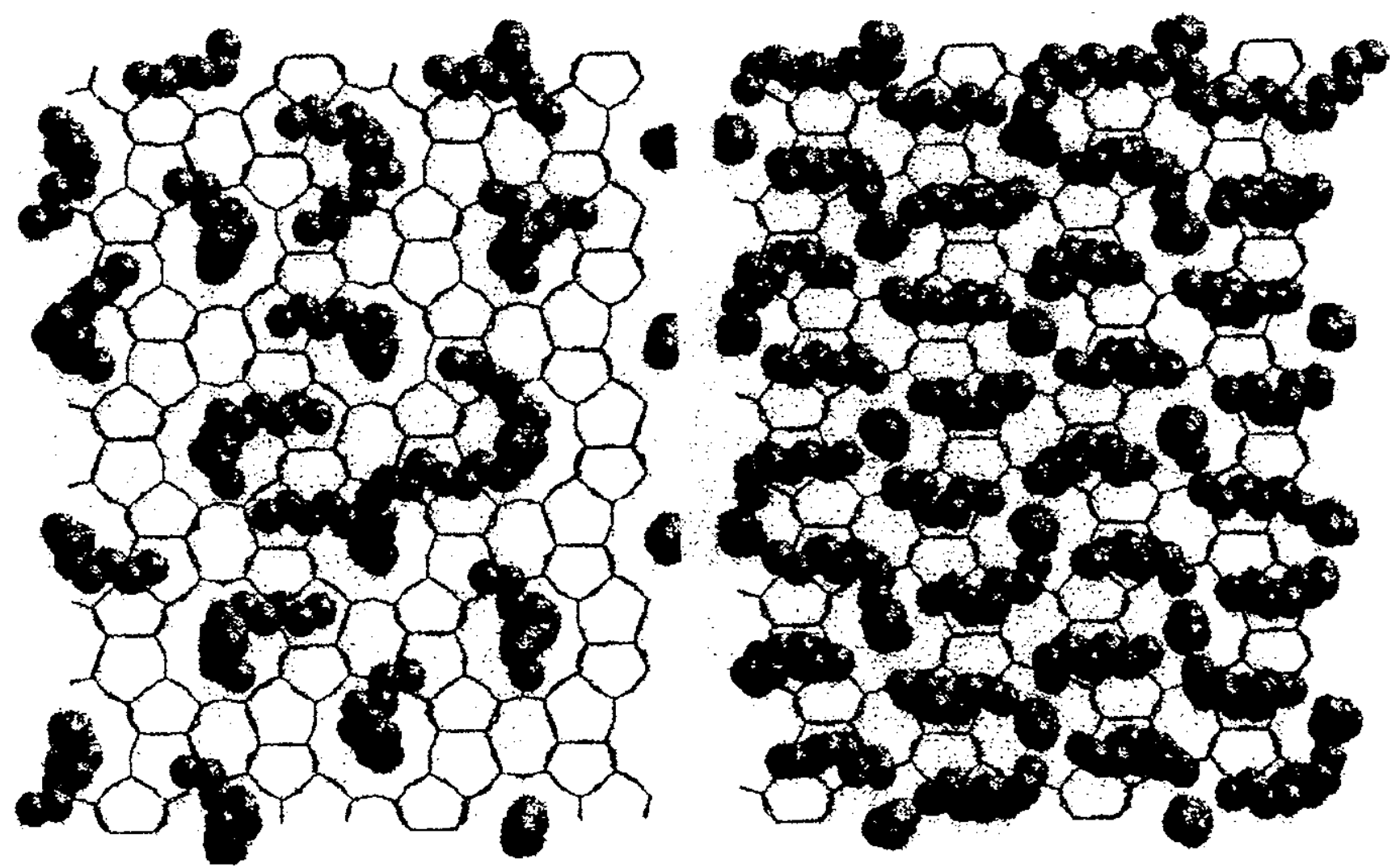

Figure 5 Hexane in silicalite: the left figure is at approximately half the maximum loading and the left figure is at almost maximum loading. The figures are projections, the zig-zag channels are in the plane of the figure and straight charnels are perpendicular to the plane of the figure. The hexane molecules are represented by gray spheres and red/orange lines represent the zeolite framework

adsorption isotherm; in order to fill the entire zeolite structure neatly, the hexane molecules located in zig-zag channels have first to be 'frozen' in these channels. This 'freezing' of the positions of the hexane molecules implies a loss of entropy and will therefore only occur if the pressure (or chemical potential) is sufficiently high to compensate for this loss. Further experimental evidence for this commensurate freezing of hexane and heptane has been found by van Well et al. ${ }^{22}$

\subsection{Phase Equilibria of $n$-Alkanes}

The Gibbs-ensemble technique was introduced by Panagiotopoulos $^{23}$ as an efficient tool to simulate vapor-liquid phase equilibria. In the Gibbs-ensemble scheme simulations of the liquid and vapor phases are carried out in parallel. Monte Carlo rules which allow for changes in the number of particles and the volume ensure that the two boxes are in thermodynamic 
equilibrium with each other. Since the two boxes are not in 'physical contact,' there is no interface and the bulk properties of the two coexisting phases can be obtained directly with a surprisingly small number of particles. This makes the Gibbs ensemble extremely efficient for phase equilibrium calculations. The major limitation of the Gibbs-ensemble technique is that one of the steps involves the exchange of particles between the two boxes. For liquids consisting of small molecules this does not cause serious problems. However, for chain molecules the probability of successful exchanges can become very small. For example, under conditions where it takes approximately $10^{3}$ attempts per successful exchange of methane, it takes of the order of $10^{3 n}$ attempts for an $n$-alkane with $n$ segments. Therefore, this technique is limited to systems containing atoms or small molecules. However, by combining the Gibbs-ensemble method with the configurational-bias Monte Carlo, the method can be made to work for much longer chain molecules.

Alkanes are thermally unstable above approximately $650 \mathrm{~K}$, which makes experimental determination of the critical point of alkanes longer than decane $\left(\mathrm{C}_{10}\right)$ extremely difficult. The longer alkanes, however, are present in mixtures of practical importance for the petrochemical industry. In these mixtures, the number of components can be so large that it is not practical to determine all phase diagrams experimentally. One therefore has to rely on predictions made by equations of state. The parameters of these equations of state are directly related to the critical properties of the pure components. Therefore, the critical properties of the long chain alkanes are used in the design of petrochemical processes, even if they are unstable close to the critical point. ${ }^{24}$ Unfortunately, experimental data are scarce and contradictory, and one has to rely on semiempirical methods to estimate the critical properties. ${ }^{24}$

Siepmann et al. ${ }^{25,26}$ have used the combination of the Gibbs-ensemble technique and configurational-bias MonteCarlo to simulate vapor-liquid equilibria of the $n$-alkanes at conditions where experiments are not (yet) feasible. Phase diagrams are very sensitive to the choice of interaction potentials. Most available models for alkanes have been obtained by fitting simulation data to experimental properties of the liquid under standard conditions. In Figure 6 the vapor -liquid curve of octane as predicted by some of these models is compared with experimental data. This figures shows that the models

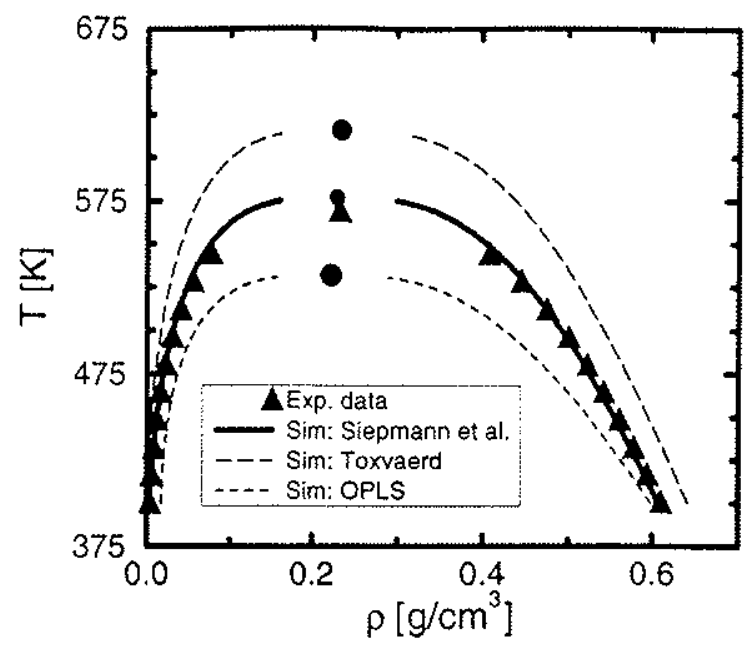

Figure 6 Vapor-liquid curve of octane: comparison of Gibbs-ensemble simulations using the OPLS model of Jorgensen and co-workers, ${ }^{27}$ the model of Toxvaerd, ${ }^{28}$ and the model of Siepmann et al. ${ }^{25,26}$

of Refs. 27 and 28 which give nearly identical liquid properties, yield estimates of the critical temperature of octane that differ by $100 \mathrm{~K}$. Siepmann et al. ${ }^{25,26}$ used these vapor-liquid equilibrium data to improve the existing models.

In Figure 7 the critical temperatures and densities as predicted by the model of Siepmann et al. are plotted versus carbon number. The simulations reproduce the experimental critical points very well. There is, however, considerable disagreement between the various experimental estimates of the critical densities.

Much of our current knowledge of the critical properties of the higher alkanes is based on extrapolations of fits of the experimental data up to $\mathrm{C}_{8}$. The most commonly used extrapolations assume that the critical density is a monotonically increasing function of the carbon number, approaching a limiting value for the very long alkanes. ${ }^{24,29}$ In contrast to these expectations, the experimental data of Anselme et al ${ }^{30}$ indicate that the critical density has a maximum for $\mathrm{C}_{8}$ and then decreases monotonically. The data of Steele (as reported in Ref. 29), however, do not give any evidence for such a maximum (see Figure 7). The simulations indicate the same trend as that observed by Anselme et al. In this context, it is
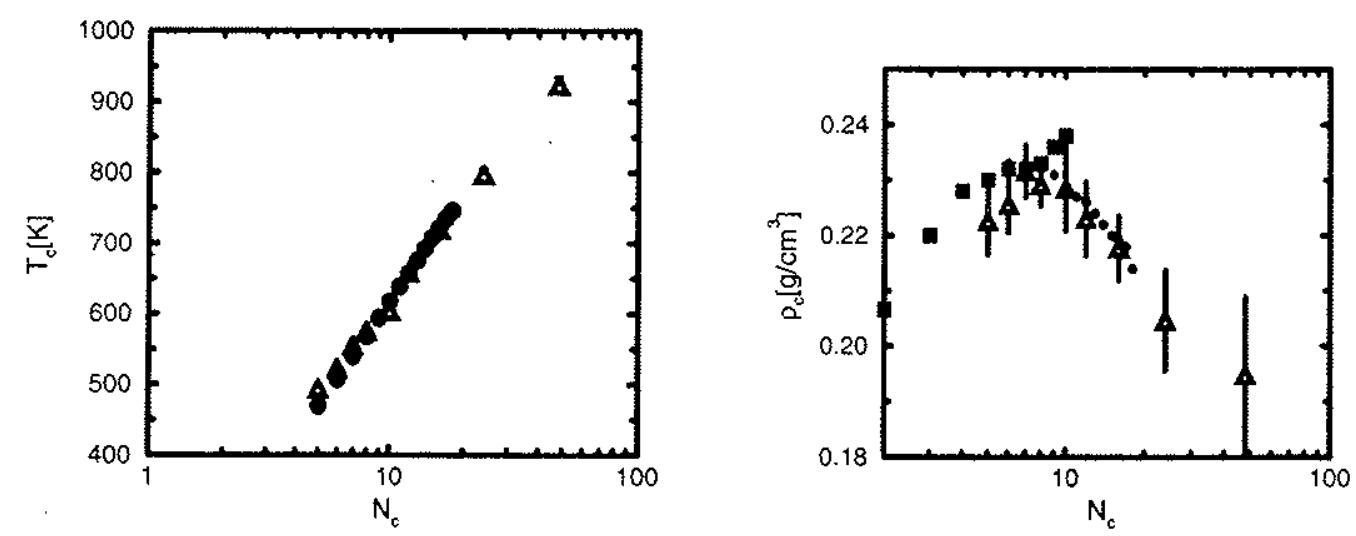

Figure 7 Critical temperature $T_{c}$ (left figure) and density $\rho_{c}$ (right figure) as a function of carbon number $N_{c}$. The open symbols are the simulation data and the closed symbols are experimental data 
interesting to note that Mooij et al. ${ }^{31}$ and Sheng et al. ${ }^{32}$ used Monte Carlo simulations to study the vapor-liquid curve of a polymeric bead spring model for various chain lengths. These studies also show a decrease of the critical density as a function of chain length. Such a decrease of the critical density with chain length is a general feature of long chain molecules, as has already been pointed out by Flory.

\subsection{Liquid Crystals}

In a liquid crystal the molecules have an orientational order but do not have translational order: a liquid crystalline state is therefore intermediate between a liquid and a solid. The simplest liquid crystalline phase is the nematic phase and most liquid crystalline materials have a nematic-isotropic phase transition (see Figure 8). Because of the applications of liquid crystals in, for example, displays, understanding the properties of these materials is of considerable practical interest. For more details on the physics and applications see Ref. 33.

In the introduction it is mentioned that short-range repulsions and long-range attractions between spherical particles are sufficient to explain the existence of the solid, liquid, and gas phases. Similarly, one can wonder what type of interactions are required to have liquid crystalline phases. More than 50 years ago, Onsager was among the first to ask this question. Onsager argued that infinitely thin hard rods have an entropy driven isotropic-nematic phase transition. Later computer simulations have shown that also other elongated hard core potentials have liquid crystalline phases (for an extensive review see Ref. 34).

Infinity thin hard rods can be considered as completely stiff polymers; completely flexible rods can be considered as ideal polymers. In contrast to thin hard rods, these ideal polymers do not have liquid crystalline phases (compare the average orientation of cooked spaghetti with uncooked spaghetti). Therefore chain flexibility is an important parameter determining whether or not a polymer has liquid crystalline behavior. Several theories have been developed to predict whether a polymer is sufficiently rigid to exhibit liquid crystalline behavior. Dijkstra and Frenkel have used computer simulations to test these theories, ${ }^{35}$

The polymer of Dijkstra and Frenkel consists of ten hard spherocylinders with a fixed bond length $l$. (A spherocylinder is a cylinder with length $L$ and diameter $D$ with hemispherical caps of diameter $D$ at each end. For suitable values of $L / D$ a fluid of (monomeric) spherocylinders has liquid-crystal phases - smectic and nematic phases.) The flexibility is determined by the bond bending potential

$$
u_{\mathrm{bb}}=\frac{C}{2 l} \theta
$$

where $\theta$ is the bond angle. The constant $C$ defines the stiffness, or persistence length of the polymer:

$$
l_{\mathrm{P}}=\frac{C}{k_{\mathrm{B}} T}
$$

For a completely flexible polymer we have $l_{p}=0$ and for $l_{\mathrm{p}} \rightarrow \infty$ the polymer is completely rigid.

Since the isotropic-nematic transition (see Figure 8) occurs at relatively high densities, the CBMC scheme provides an efficient scheme for generating new configurations of the polymers. The results of the simulations are shown in Figure 9. For various persistence lengths Dijkstra and Frenkel have calculated the coexistence densities of the isotropic and nematic phases. In addition, comparison with theoretical predictions of this transition shows that although the theories are qualitatively correct the description of the nematic phase needs significant improvements before quantitative agreement can be obtained.
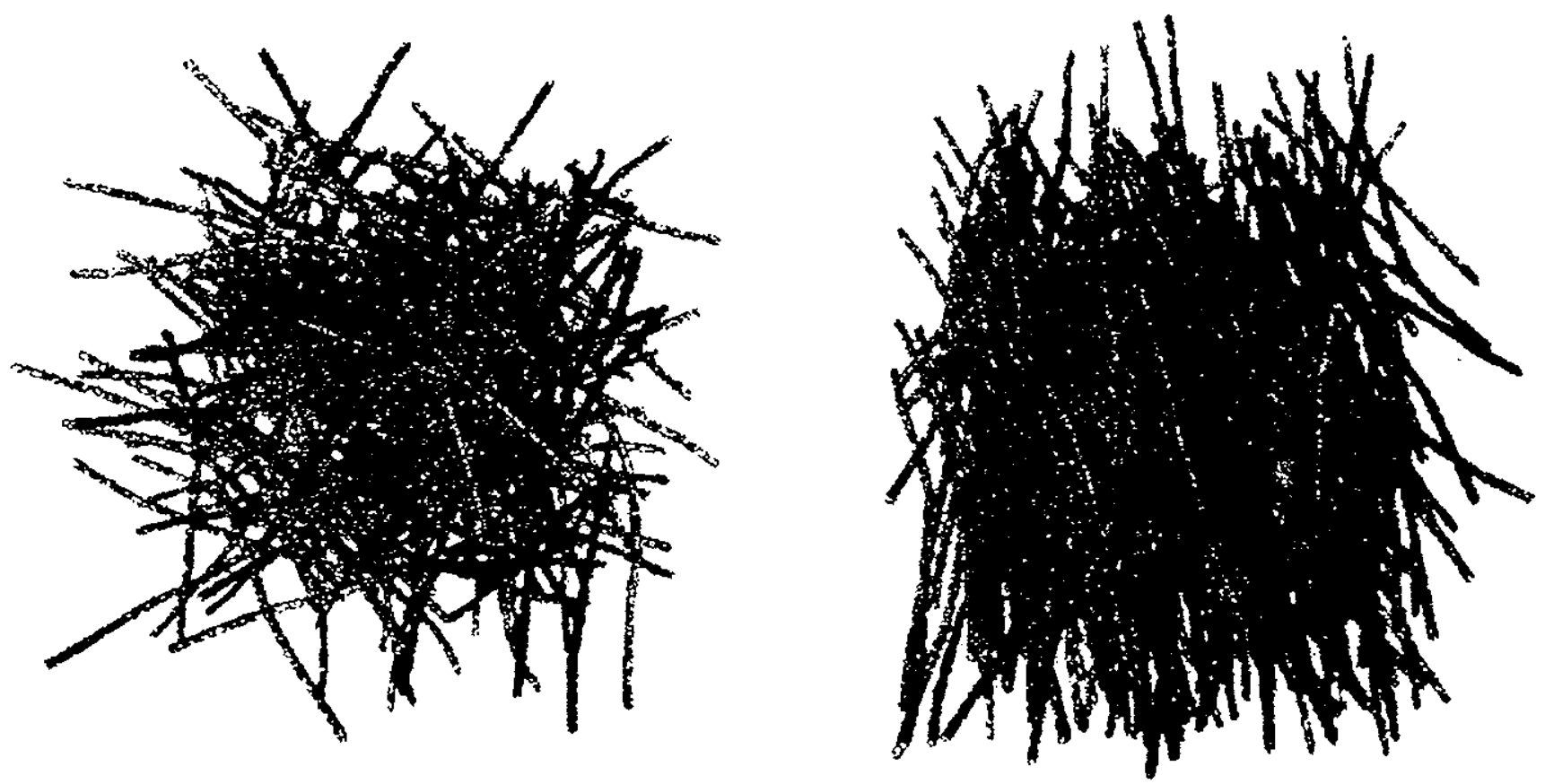

Figure 8 Isotropic-nematic phase transition, in the smectic phase (right) the molecules have orientational order but do not show any long-range translational order. In the isotropic phase (left) the orientational order is lost (Figure by M. Dijkstra) 


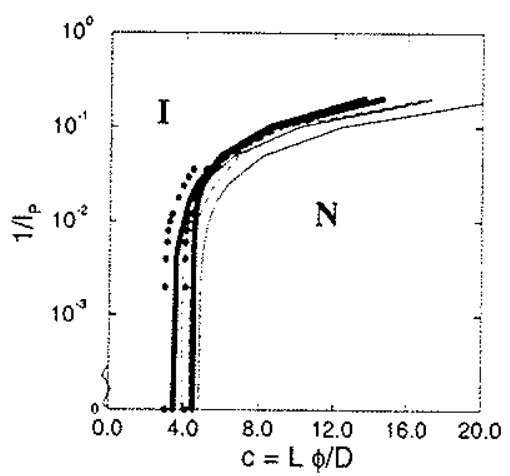

Figure 9 The isotropic-nematic coexistence densities $\phi$ of flexible polymers for various persistence lengths $l_{p}$. The lines are the predictions of various theories and the dots the results of computer simulations

\subsection{Mixtures of Colloids and Polymers}

Thus far, the configurational-bias Monte Carlo scheme has been presented exclusively as a method to generate conformations of chain molecules. In fact, the method is more general than that. It can be used as a scheme to perform collective rearrangements of any set of labeled coordinates. In fact, the scheme can be used to carry out Monte Carlo moves to swap $n$ small particles within a volume $\Delta \mathrm{V}$ with one large particle that occupies the same (excluded) volume. This application of the CBMC scheme has been exploited by Biben ${ }^{36}$ to study mixtures of large and small hard spheres. Gibbs-ensemble simulations of mixtures of spherical colloids and rod-like polymers were performed by Bolhuis and Frenkel ${ }^{37}$ using CBMC-style particle swaps and a closely related approach was employed by Dijkstra ${ }^{38,39}$ to study phase separation in mixtures of large and small hard-core particles on a lattice.

Bolhuis and Frenkel are interested in simulating the properties of colloidal solutions. Examples of such solutions are milk, paint, or mayonnaise. Since a single colloidal particle may contain over $10^{9}$ atoms: it is not possible to model such a particle as a collection of atoms. However, it is possible to describe colloidal solutions using coarse-grained models. For example, a suspension of silica spheres can be described surprisingly accurately with a hard-sphere potential. Similarly to the hard-sphere fluid, such a colloidal suspension has a fluid-solid transition, but not a liquid-gas transition. Experimentally, it is observed that a liquid-gas transition can be induced by adding polymers to the suspension.

The effect of adding polymers is like having attractive interactions between the colloidal particles. These effective interactions occur even in systems that have only excluded volume interactions and are therefore called entropic interactions. To see this, consider a system of two colloidal particles to which we add a polymer. We assume that this polymer behaves like an ideal polymer except that it cannot overlap with the colloidal particle, that is, the polymer and colioid have excluded volume interactions. If the distance between the colloids is large, the total entropic contribution of the polymer is related to the total number of configurations of the polymer minus those configurations that overlap with the first colloidal particle and those that overlap with the second. When the distance between the two colloidal particles is smaller than the length of the polymer, the total entropy increases. If, for such a system, we were to compute the entropy by subtracting those configurations of the polymer that overlap with the two colloids, we would count those configurations twice which overlap with both colloids at the same time. This increase of the entropy as two colloidal particles approach each other gives rise to an effective attractive force between the particles. Similar effects can be expected in mixtures of infinitely thin hard rods and hard spheres. If these rods are sufficiently long, they may induce a vapor-liquid like transition for the hard spheres.

Bolhuis and Frenkel have studied the phase behavior of a mixture of hard spheres and hard rods. In particular, Bolhuis and Frenkel used Gibbs-ensemble simulations to determine the 'vapor-liquid' coexistence curve. In a Gibbs-ensemble simulation one simulates two boxes that are kept in equilibrium with each other via Monte Carlo rules. In this case the 'gas' box has as a low density of hard spheres and the 'liquid' box has a high density of spheres. Similarly to the phase equilibrium calculation of linear alkanes, the exchange step, in which particles are exchanged between the two boxes, is the bottleneck of the simulation. For example, the insertion of a sphere into the 'gas phase' would almost always fail because of overlaps with some of the rods. Bothuis and Frenkel have used the following scheme to make this exchange possible:

1. Select a sphere in one of the boxes at random and insert this sphere at a random position in the other box.

2. Remove all the rods that overlap with this sphere. These rods are inserted in the other box. If these rods were inserted at random, almost always one of the rods would overlap with a sphere and such a move would be rejected. However, if one tries several orientations and positions of the rods and selects an acceptable configuration using the configurational-bias Monte Carlo scheme one can make such a move possible. During the insertion of the rods one has to be careful that a detailed balance is obeyed (for details see Ref. 37).

Because of this redistribution scheme of the rods during the insertion step using the CBMC one can generate configurations that do not have overlap. This allowed Bolhuis and Frenkel to exchange spheres between the two boxes and obtain the coexistence densities. The result of these Gibbs-ensemble simulations is shown in Figure 10. The figure shows that if one increases the fugacity ${ }^{4}$ (chemical potential) of the rods and hence the concentration of rods, a demixing occurs in a phase with a low density of spheres and in a phase with a high density of spheres. (Experimental colloidal systems are usually in open contact with a reservoir of rod-like particles, therefore Bolhuis and Frenkel used the fugacity instead of the pressure.) If the rods are longer, this demixing occurs at a lower concentration. Important to note is that in this system the particles interact with hard core interactions only. Therefore this demixing is driven by entropy.

An interesting extension of this technique used by Bolhuis and Frenkel would be to biological systems. For example, if one would like to make a large displacement of a part of a protein, in vacuum this would be relatively easy to do. In an aqueous solution, however, one has to remove the water molecules that overlap with that part of the protein that has been moved and the water molecules need to be redistributed. This redistribution can be done with the scheme of Bolhuis and Frenkel. 


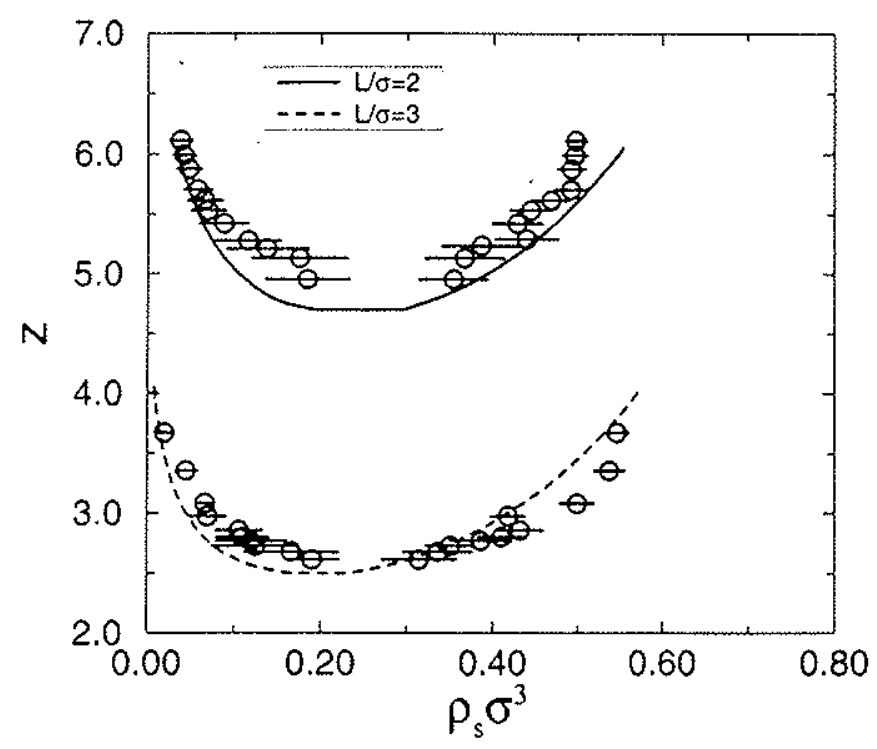

Figure 10 Coexistence curves for a mixture of hard spheres and rods as obtained by Bolhuis and Frenkel. The $x$-axis gives the density and the $y$-axis the fugacity (chemical potential) of the rods. $L / \sigma$ is the ratio of the length of the rod and the diameter of the hard sphere

\subsection{Parallel Monte Carlo Simulations}

A completely different application of the CBMC ideas is used by Esselink et al. ${ }^{40}$ to develop an algorithm to perform Monte Carlo simulations on parallel computers

When the size of the simulated system is necessarily large or the simulation times are long, one would like to use as much computing power as possible, for instance by using more than one processor in parallel. This concept of increasing computing power by placing processors in parallel is not new. Due to physical constraints, there is a theoretical speed limit beyond which no single processor will ever operate. The only way to build faster computers still is by placing them in parallel. Nowadays, there is a wide variety of vendors selling parallel computers. The products differ in processor technology, in the way communication between processors is handled, and in the level of abstraction offered to the user or programmer. Some users don't want to 'think parallel,' but rather prefer the original sequential view of programming. In that case, a sophisticated compiler, operating system or specific hardware, needs to be able to cope with the parallel architecture. Indeed, automatic parallelization is a large research area. However, 'hand-coding' of parallelism so far often yields the best results.

The molecular dynamics technique is well suited for parallel computations, since the necessary computations are the same for all particles. We can use this natural parallelism if during the computation each processor calculates the trajectories of all particles that are assigned to this processor. An alternative would be not to distribute parts of the simulation box, but to assign particles to processors. However, this would imply communication between every pair of processors, in order to look for close atoms. This is known to perform poorly for large simulation systems and processor networks.

Whereas molecular dynamics is very well suited for parallelism, parallel Monte Carlo appears to be a contradiction

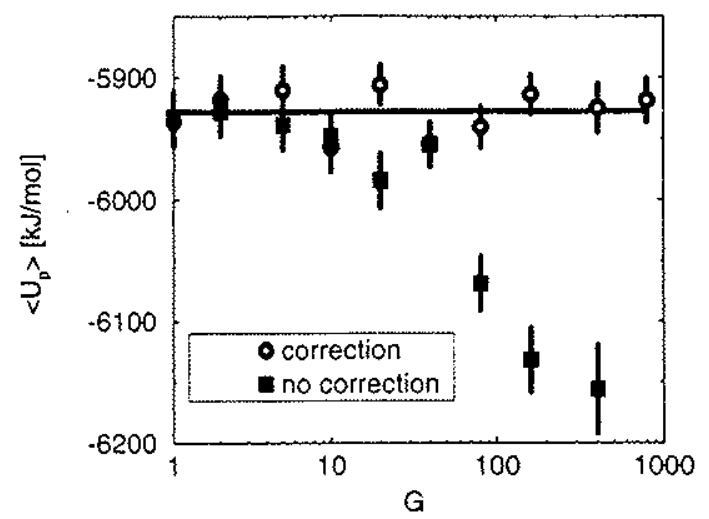

Figure 11 The average energy of an alkane molecule in a zeolite as a function of the number of processors. If the acceptance rules are not corrected, a bias (dependence on the number of processors) in the results is found ${ }^{40}$

in terms. Since the Monte Carlo procedure is an intrinsically sequential process, one has to know whether the current move is accepted or rejected before one can continue with the next move. The conventional way of introducing parallelism is to distribute the energy calculation over various processors, or to farm out the calculation by performing separate simulations over various processors. Although the last algorithm is extremely efficient and requires minimum skills to use a parallel computer, it is not a truly parallel algorithm. For example, farming out a calculation is not very efficient if the equilibration of the system takes a significant amount of cpu time.

In the algorithm of Esselink et al. several trial positions are generated in parallel and out of these trial positions the one with the highest probability of being accepted is selected with the highest probability. This selection step introduces a bias which is removed by adjusting the acceptance rules. The generation of each trial move, which includes the calculation of the energy (or Rosenbluth factor in the case of chain molecules), is distributed over the various processors. In Figure 11 the results of a typical simulation are shown, in this case the average energy of a molecule as a function of the number of processors of the computer. If the calculation is done correctly the result should, of course, be independent of the number of processors. The figure shows a bias in the results, i.e., a dependency on the number of processors occurs if the acceptance rules are not corrected. This example illustrates the importance of adjusting the acceptance rule; if the acceptance rule is not used systematic errors are made.

\section{CONCLUDING REMARKS}

In this article we have discussed the configurational-bias Monte Carlo scheme. In this method we combine tailor-made algorithms to generate new configurations with an appropriate acceptance rule. These acceptance rules are chosen so that they remove a possible bias in the generation of these new configurations. Here we have introduced this scheme in the context of simulating chain molecules. Some examples are dis. cussed in which this technique is many orders of magnitude more efficient than the conventional Monte Carlo techniques or molecular dynamics. 


\section{RELATED ARTICLES}

Intermolecular Interactions by Perturbation Theory; Molecular Dynamics and Hybrid Monte Carlo in Systems with Multiple Time Scales and Long-range Forces: Reference System Propagator Algorithms; Molecular Dynamics: Simulations of Nucleic Acids; Molecular Dynamics: Studies of Lipid Bilayers; Molecular Dynamics: Techniques and Applications to Proteins; Monte Carlo Simulations for Liq. uids; Monte Carlo Simulations for Polymers.

\section{REFERENCES}

1. G. Ciccotti, D. Frenkel, and I. R. McDonald, 'Simulation of Liquids and Solids: Molecular Dynamics and Monte Carlo Methods in Statistical Mechanics', North-Holland, Amsterdam, 1987

2. J. M. Thomas, Sci. Am., 1992, 266, 82-88.

3. C. R. A Catlow ed., 'Modelting of Structure and Reactivity in Zeolites', Acadernic, London, 1992.

4. R. L. June, A. T. Bell, and D. N. Theodorou, J. Phys. Chem. $1992,96,1051-1060$.

5. E. Hemandez and C. R. A. Catlow, Proc. R. Soc. Lond. A, 1995 , 448, $143-160$.

6. S. J. Goodbody, K. Watanabe, D. MacGowan, J. P. R. B. Walton, and N. Quirke, J. Chem. Soc., Faraday Trans., 1991, 87, $1951-1958$

7. J. I. Siepmann and D. Frenkel, Mol. Phys., 1992, 75, 59-70.

8. M. N. Rosenbluth and A. W. Rosenbluth, J. Chem. Phys., 1955, 23, 356-359.

9. J. Harris and S. A. Rice, J. Chem. Phys, 1988, 88, 1298-1306.

10. D. Frenkel, G. C. A. M. Mooij, and B. Smit, J. Phys. Condens. Matter, 1992, 4, 3053-3076.

11. J. J. de Pablo, M. Laso, and U. W. Suter, J. Chem. Phys., 1992, 96, 2395-2403

12. J. Batoulis and K. Kremer, J. Phys. A. Math. Gen., 1988, 21, $127-146$.

13. D. Frenkel and B. Smit, 'Understanding Molecular Simulations: from Algorithms to Applications', Academic Press, San Diego, 1996.

14. B. Smit and J. I. Siepmann, J. Phys. Chem, 1994, 98, $8442-8452$.

15. B. Smit and J. I. Siepmann, Science, 1994, 264, 1118-1120.

16. E. J. Maginn, A. T. Bell, and D. N. Theodorou, J. Phys. Chem., $1995,99,2057-2079$.

17. B. Bigot and V. -H. Peuch, J. Phys. Chem., 1995, 99 8206-8215.

18. R. Evans, in 'Liquides aux Interface/Liquids at Interfaces', Les Houches, Session XLVIII, 1988, eds. J. Charvolin, J. F. Joanny, and J. Zinn-Justin, North Holland, Amsterdam, 1990, p. I-98.

19. S. J. Gregg and K. S. W. Sing, 'Adsorption, Surface Area and Porosity', Academic Press, London, 1982.

20. H. Stach, U. Lohse, H. Thamm, and W. Schirmer, Zeolites, 1986, 6, 74-90.

21. B. Smit and T. L. M. Maesen, Nature, 1995, 374, 42-44.

22. W. J. M. van Well, J. P. Wolthuizen, B. Smit, J. H. C. vanHooff, and R. A. van Santen, Angew. Chem., Int. Ed. Engl., $1995,34,2543-2544$

23. A. Z. Panagiotopoulos, Mol. Phys., 1987, 61. 813-826

24. C. Tsonopoulos, AIChE J., 1987, 33, 2080-2083.

25. J. 1. Siepmann, S. Karaborni. and B. Smit. Nature, 1993, 365, $330-332$.

26. B. Smit, S. Karaborni, and J. I. Siepmann, J. Chem. Phys., 1995. 102, 2126

27. W. L. Jorgensen, J. D. Madura, and C. J. Swenson, J. Am. Chem. Soc., 1984, 106, 6638-6646.

28. S. Toxvaerd, J. Chem. Phys, 1990, 93, 4290-4295.
29. C. Tsonopoulos and Z. Tan, Fluid Phase Equilibria, 1993, 83, $127-138$.

30. M. J. Anselme, M. Gude, and A. S. Teja, Fluid Phase Equilibria, 1990, 57, 317-326.

31. G. C. A. M. Mooij, D. Frenkel, and B. Smit, J. Phys. Condens. Matter, 1992, 4, L255-L259.

32. Y. -J. Sheng, A. Z. Panagiotopoulos, S. K. Kumar, and I. Szleifer, Macromolecules, 1994, 27, 400-406.

33. P. G. de Gennes and J. Prost, "The Physics of Liquid Crystals", 2nd edn., Clarendon, Oxford, 1995.

34. M. P. Allen, G. T. Evans, D. Frenkel, and B. M. Mulder, in 'Advances in Chemical Physics', eds., I. Prigogine and S. A. Rice, Wiley, New York, 1993, Vol. LXXXV1.

35. M. Dijkstra and D. Frenkel, Phys. Rev. E, 1995, 51, $5891-5898$.

36. T. Biben, Ph.D. Thesis, Université Claude Bernard, Lyon, 1993

37. P. Bolhuis and D. Frenkel, J. Chem. Phys., 1995, 101, 9869-9875

38. M. Dijkstra and D. Frenkel, Phys. Rev. Lett., 1994, 72, 298-300.

39. M. Dijkstra, D. Frenkel, and J. -P. Hansen, J. Chem. Phys. 1994, 101, 3179-3189.

40. K. Esselink, L. D. J. C. Loyens, and B. Smit, Phys. Rey. E, $1995,51,1560-1568$.

\section{Monte Carlo Simulations for Liquids}

William L. Jorgensen

Yale Universiry, New Haven, CT, USA

\section{Background}

2 Potential Functions and Their Evaluation

3 Properties of Liquids and Their Convergence

4 Water

5 Organic Liquids

6 Conclusion

7 Related Articles

8 References

\section{BACKGROUND}

Monte Carlo (MC) methods are used in many fields to provide numerical solutions to multi-dimensional integrals by random sampling of the integration variables. For manyparticle systems in classical statistical mechanics, the problem focuses on solving the configurational integral that appears in the averages for a property $Q$ (equations 1 and 2 ). ${ }^{1}$

$$
\begin{aligned}
\langle Q\rangle & =Q_{k}+\int Q(\mathbf{X}) P(\mathbf{X}) \mathrm{d} \mathbf{X} \\
P(\mathbf{X}) & =\exp (-\beta E(\mathbf{X})) / \int \exp (-\beta E(\mathbf{X})) \mathrm{d} \mathbf{X}
\end{aligned}
$$

These equations are for the canonical NVT (constant number of particles, volume, and temperature) ensemble where $P(\mathbf{X})$ is the Boltzmann factor, $E(\mathbf{X})$ is the total potential energy, $\beta=$ $1 / k_{\mathrm{B}} T$, and the integrals are taken over all possible geometrical 